\title{
Factores de riesgo para infección del tracto urinario asociado al uso de catéter urinario permanente en pacientes adultos hospitalizados
}

\author{
Risk factors for urinary tract infection associated with the use of urinary catheters in hospitalized adult \\ patients
}

Elena Véliz y Teresa Vergara'

'Unidad de Infecciones Asociadas a la Atención en Salud. Hospital Militar de Santiago.

Los autores declaran no tener conflicto de interés. No tuvo fuente de financiamiento.

Recibido: 8 de diciembre de 2019 / Aceptado: 5 de octubre de 2020

\section{Resumen}

La infección del tracto urinario asociada al catéter urinario permanente (ITU/CUP) es un problema relevante en los centros de salud por su alta frecuencia. Objetivo: Identificar factores de riesgo de ITU en pacientes adultos con CUP. Material y Método: Estudio caso control efectuado entre los años 2010-2016 en el Hospital Militar de Santiago. Se realizó un análisis estadístico univariado y multivariado por medio del modelo de regresión logística binaria, con variables como edad, duración e indicación del dispositivo, algunas comorbilidades y la instalación del dispositivo luego de 15 días hospitalización. Resultado: Se obtuvo un total de 63 casos y 123 controles. Fueron variables predictoras de ITU/CUP la duración del CUP desde el séptimo día en adelante (OR 2,6 IC 1,4-4,9, p = 0,004) y la instalación del CUP con una estadía de hospitalización previa de 15 días y más (OR 7,8 IC 2,9$20,9 \mathrm{p}=0,000)$. No se encontró asociación con la edad mayor de 80 años, comorbilidades como diabetes y vejiga neurogénica e indicación de instalación. Conclusiones: Los resultados permiten focalizar las intervenciones, evaluando la necesidad real de indicación de CUP en pacientes con estadía hospitalaria previa de dos semanas y fomentar el retiro de CUP antes del séptimo día de uso.

Palabras clave: infección del tracto urinario; catéter urinario permanente; factores de riesgo; infecciones asociadas a la atención en salud.

\section{Introducción}

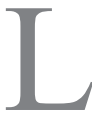

a infección del tracto urinario asociada al catéter urinario permanente (ITU/CUP) es un problema relevante en los centros de salud, especialmente en las unidades de cuidados intensivos ${ }^{1,2}$,

\begin{abstract}
Catheter-associated urinary tract infection (CAUTI) is a relevant problem in health centers because of its high frequency. Objective: To identify UTI risk factors in adult patients with urinary catheter. Material and Method: Control case study carried out between the years 2010-2016 at the Military Hospital of Santiago, Chile. Univariate and multivariate statistical analysis were performed using the binary logistic regression model, variables such as age, duration and indication of the device, some comorbidities and the installation of the device after 15 days of hospitalization. Result: A total of 63 cases and 123 controls were obtained. Predictive variables of CAUTI for the duration of the urinary catheter from the 7th day onwards (OR 2.6 IC $1.4-4.9, p=0.004)$ and the installation of the urinary catheter with a previous hospital stay of 15 days and more (OR 7.8 CI 2.9-20.9 p = 0.000 ). No association was found in age over 80 years, comorbidities such as diabetes and neurogenic bladder and indication. Conclusions: The results permitted to focus the interventions, evaluating the real need for indication of CUP in patients with previous hospital statistics of 2 weeks and encouraging the withdrawal of CUP before the 7 th day of use.

Keywords: urinary tract infection; permanent urinary catheter; risk factors; healthcare-associated infections.
\end{abstract}

ya que la mayoría de los pacientes requiere un dispositivo de este tipo dada su condición crítica.

Se estima que la ITU/CUP representa entre 30 y $40 \%$ de las ITU adquiridas en el hospital. El 25\% de los pacientes hospitalizados en servicios generales y cerca de $89 \%$ de aquellos en unidades críticas 
tiene un $\mathrm{CUP}^{1,3} \mathrm{y}$ una parte de estos pacientes desarrollan bacteriuria, como antesala de la infección, dependiendo del tiempo de duración del cateterismo. La mortalidad atribuible de esta infección es de $13 \%$, especialmente cuando se asocian a bacteriemias por enterobacterias multirresistentes ${ }^{4}$.

En Chile, de acuerdo al informe de vigilancia de infecciones asociadas a la atención de salud (IAAS) 2017 del Ministerio de Salud de Chile (Minsal) ${ }^{5}$, las ITU corresponden a la infección más frecuente en hospitales de cualquier complejidad y es un indicador obligatorio a vigilar en pacientes adultos que tienen el dispositivo por más de 24 horas.

Los factores de riesgo descritos para desarrollar una ITU pueden ser modificables y no modificables. Entre los primeros, destaca la inserción del dispositivo urinario en el quirófano ${ }^{6}$, la duración del catéter con permanencias superiores a seis días, inclusive 14 días. Se plantea que pacientes ingresados para procedimientos quirúrgicos mayores con una duración del CUP por más de dos días tienen mayor riesgo de desarrollar ITU ${ }^{7}$. Otro factor de riesgo modificable corresponde a la indicación médica incorrecta de instalación de CUP y al incumplimiento de la técnica aséptica en la instalación y mantención del dispositivo. Dentro de los factores de riesgo no modificables se incluye la inserción del CUP después del sexto día de hospitalización o después del $15^{\circ}$ día y la edad también se asocia a un mayor riesgo en pacientes mayores de 60 y 90 años $^{7-10}$. También se incluyen las anomalías del tracto urinario, la insuficiencia renal crónica ${ }^{6}$, diabetes mellitus, vejiga neurogénica, pacientes con creatinina sérica mayor de $2 \mathrm{mg} / \mathrm{dl}^{7,8}$, pacientes urológicos y aquellos con incontinencia fecal ${ }^{11,12}$.

Debido a que los factores de riesgo pueden tener variaciones entre diferentes áreas geográficas en el mundo o entre países desarrollados y no desarrollados, se hace necesario identificar qué factores de riesgo pueden estar participando en nuestra realidad local. El objetivo de esta investigación fue identificar los factores de riesgo de ITU/CUP en pacientes adultos y para ello se decidió explorar factores de riesgo modificables y no modificables que permitan orientar futuras estrategias de intervención.

\section{Material y Método}

Estudio caso control con pareo 1:2. La selección de casos se realizó mediante la revisión de los registros de vigilancia epidemiológica del programa de control de infecciones del establecimiento para el periodo 2010-2016. Se seleccionaron los pacientes con ITU/CUP de acuerdo a las definiciones del Minsal ${ }^{13}$. Se incluyeron solo pacientes adultos correspondientes a los servicios de UCI, UTI, UCI cardiovascular, medicina y cirugía; solo se consideraron casos nuevos y se excluyeron los pacientes urológicos.

La obtención de los controles se realizó mediante la selección aleatoria de pacientes hospitalizados durante el mismo periodo que los casos (2010-2016) que estuvieron expuestos al CUP pero que no cursaron con ITU. Estos se parearon por servicio, sexo y mes de hospitalización, siempre corroborando que los controles no calificaran como caso.

La búsqueda de información respecto de las variables se realizó a través de la revisión de la ficha clínica de los pacientes.

\section{Variables independientes incorporadas en el estudio de factores de riesgo para ITU/CUP}

Se realizó un análisis estadístico univariado a través de la prueba de $\chi^{2}$ o prueba exacta de Fisher de todas las variables planteadas (Tabla 1 ).

Esto permitió obtener un odds ratio (OR) para conocer

Tabla 1. Variables independientes incorporadas en el estudio de factores de riesgo para ITU/CUP

Variable

Edad

Duración del CUP

Comorbilidades

Indicación del CUP

\section{Descripción}

80 años y más

Siete días y más

Diabetes mellitus, vejiga neurogénica

Retención urinaria aguda u obstrucción vesical, medición urinaria en paciente críticamente enfermo, procedimientos quirúrgicos seleccionados, cirugía urológica o cirugías en estructuras contiguas al tracto genitourinario, cirugía de duración prolongada, probable recepción de altos volúmenes de infusión o diuréticos durante la cirugía, monitoreo intraoperatorio, heridas sacras o perineales abiertas, inmovilización prolongada (tórax inestable o zona lumbar inestable), medida de confort en pacientes con medidas de sostén, paciente con cateterismo intermitente mayor a cuatro veces en el día 1,7,14

Días de instalación del CUP postingreso

Instalación del CUP a los 15 días de hospitalización del paciente y más 
la asociación entre las variables independientes y la variable dependiente. Se consideró como un resultado estadísticamente significativo un valor de $\mathrm{p}=0,05$ o menor para cada variable independiente. Para la variable días de instalación de CUP antes de la infección, se exploraron otros intervalos también en forma dicotómica para precisar el rango de mayor riesgo. También se realizó un análisis multivariado para identificar factores independientes y predecir el grado de correlación de las covariables con ITU/CUP. Se utilizó el modelo de regresión logística binaria, el análisis estadístico se realizó con SPSS.

\section{Resultados}

En el período estudiado se obtuvo un total de 63 pacientes con ITU/CUP, en donde la totalidad categorizaba como casos; se pudo obtener un total de 123 controles debido a que tres pacientes tuvieron solo un control (pacientes de UCI coronaria), debido al bajo índice ocupacional y escasa indicación de CUP en el período.

Las características demográficas y los resultados del análisis univariado se presentan en la tabla 2, destacando que la variable edad de 80 años y más no mostró ser un factor de riesgo (OR 1,3 IC 0,7-2,4, p=0,4) para desarrollar ITU/CUP. Asimismo, se evaluó en forma complementaria el riesgo en pacientes de 60 años y más y de 50 años y más, obteniendo resultados similares.

En la variable duración del CUP, la permanencia por siete días o más mostró una asociación de riesgo para ITU/CUP (OR 2,6 IC 1,4-4,9, p = 0,004); sin embargo, este riesgo se demostró desde el cuarto día de CUP (OR 2,4 IC 1-5,6 p =0,03), por el contrario, el tercer día de mantención no demostró riesgo. La instalación del CUP luego de 15 días de hospitalización del paciente se asoció a ITU/CUP (OR 7,8 IC 2,9-20,9 p =0,000), no obstante, la instalación a los 10 días no mostró mayor riesgo (Tabla 2).

Respecto de las comorbilidades estudiadas, la presencia de diabetes mellitus y vejiga neurogénica no mostraron ser factores de riesgo. En cuanto la indicación apropiada del dispositivo, $88 \%$ de los CUP en el grupo de casos y en $90 \%$ de los controles estaba bien indicado (en base a criterios definidos por la evidencia científica), sin diferencias significativas. En ambos grupos la incontinencia urinaria fue la causa más frecuente de indicación inapropiada.

Debido a que más de una variable apareció asociada al riesgo, se efectuó un análisis multivariado a través del método de regresión logística binaria, el cual demostró que las variables duración del CUP $\geq$ siete días (OR 1,9 IC 95 1,0-3,9) y hospitalización previa a la instalación del CUP $\geq 15$ días (OR 6,6 IC 95 2,2-19,1) estuvieron asociadas en forma independiente al riesgo de ITU/CUP. De esta manera estas variables parecen ser predictoras de ITU/CUP (Tabla 3).

\section{Discusión}

En este estudio se evaluaron varios factores de riesgo para desarrollo de ITU/CUP, demostrando que la permanencia del catéter mayor a siete días y la instalación del dispositivo urinario luego de 15 días o más de hospitalización, están asociados al riesgo en forma independiente y de manera significativa.

\section{Duración del CUP}

De acuerdo a la revisión de la evidencia científica se planteó como factor de riesgo la mantención del CUP por siete o más días, considerando que se adiciona entre 3 y $10 \%$ de riesgo de infección por cada día de cateterismo 9 . Así nuestros resultados son congruentes con lo descrito en la literatura especializada; sin embargo, es importante destacar que nuestro estudio plantea que el riesgo aumenta desde el cuarto día de mantención del CUP $(\mathrm{p}<0,05)$. Savas y cols. llegaron a la misma conclusión, planteando que lo más importante es evitar el cateterismo innecesario y que el CUP debe ser retirado tan pronto sea posible ${ }^{15}$, de ahí la relevancia de evaluar diariamente la necesidad del dispositivo. Es así como en un estudio de cohorte se observó que $8,5 \%$ de los CUP no tenían indicación al séptimo día y que aumentaba a $16,3 \%$ el día 14 y a $23,8 \%$ el día $23^{16}$. En otro estudio efectuado en un hospital universitario de Michigan, se les preguntó a los médicos y estudiantes de medicina cuál de sus pacientes tenía un catéter urinario, se observó que $28 \%$ de los entrevistados desconocían si sus pacientes tenían CUP o no y en $31 \%$ de los pacientes el cateterismo no estaba bien justificado. Además, las indicaciones médicas para la instalación del CUP solo se pudieron verificar en la mitad de los pacientes ${ }^{17}$.

Los programas de intervención centrados en la instalación y retiro oportuno a través de la educación y sistemas de alerta han demostrado efectividad en la reducción de tasa de ITU/CUP y también en los costos por este concepto $^{18}$. Bell y cols. implementaron sistemas de alertas electrónicas en todos los pacientes que tenían CUP y diariamente el médico debía contestar a la pregunta si el dispositivo debía mantenerse o no, y si la respuesta era afirmativa este debía marcar algunas de las alternativas de indicación para este dispositivo. Además, se integró la educación en indicaciones de uso de CUP para enfermeras y médicos, logrando una reducción del $40 \%$ de días de uso de CUP en un periodo de tres años y de la tasa infección de 4 a 0 por 1.000 días $^{19}$. Un meta-análisis logró demostrar que la tasa de ITU/CUP se redujo en $52 \%$ ( $p \leq$ a 0,001$)$ con el uso de recordatorios o una orden de suspensión del CUP. Además, se redujo la duración media del cateterismo en un $37 \%$, lo que resultó en 2,6 días menos de uso de este dispositivo por paciente en los grupos de intervención versus el control ${ }^{20}$. Una revisión sistemática respecto de las mejores estrategias en prevención de ITU/CUP logró 


\begin{tabular}{|c|c|c|c|c|}
\hline Variable & Casos & Controles & OR (IC 95) & Valor $p$ \\
\hline $\mathrm{N}$ & 63 & 123 & & \\
\hline Edad promedio en años (rango) & $71,2(21-97)$ & $71,8(22-105)$ & & NS \\
\hline Género femenino n (\%) & $37(58,7)$ & $72(58,5)$ & & NS \\
\hline Género masculino n (\%) & $26(41,3)$ & $51(41,5)$ & & NS \\
\hline \multicolumn{5}{|l|}{ Factores de riesgo } \\
\hline Edad $\geq 80$ años & $27(42,8)$ & $117(95)$ & $1,3(0,7-2,4)$ & 0,4 \\
\hline \multicolumn{5}{|l|}{ Duración del catéter } \\
\hline Catéter urinario $\geq 7$ días & $41(65)$ & $22(17,8)$ & $2,6(1,4-4,9)$ & $0,003 *$ \\
\hline Catéter urinario igual a 6 días & $43(68,2)$ & $64(52)$ & $1,9(1,0-3,7)$ & $0,04^{*}$ \\
\hline Catéter urinario igual a 5 días & $49(77,7)$ & $75(60,9)$ & $2,2(1,1-4,5)$ & $0,02^{*}$ \\
\hline Catéter urinario igual a 4 días & $55(87,3)$ & $91(73,9)$ & $2,4(1-5,6)$ & $0,03^{*}$ \\
\hline Catéter urinario igual a 3 días & $60(98,4)$ & $122(99,1)$ & $0,1(0,01-1,6)$ & 0,1 \\
\hline \multicolumn{5}{|l|}{ Estadía previa } \\
\hline$\geq 15$ días de hospitalización previa a instalación de CUP & $18(28,5)$ & $6(4,8)$ & $7,8(2,9-20,9)$ & $0,000^{*}$ \\
\hline \multicolumn{5}{|l|}{ Comorbilidades } \\
\hline Diabetes & $20(34,9)$ & $30(24,3)$ & $1,2(0,6-2,5)$ & 0,6 \\
\hline Vejiga neurogénica & $4(6,3)$ & $9(7,3)$ & $2,9(0,6-13,9)$ & 0,22 \\
\hline \multicolumn{5}{|l|}{ Criterios de indicación CUP } \\
\hline Cumple criterios & $56(88,8)$ & $111(90,2)$ & $1,5(0,8-2,9)$ & 0,18 \\
\hline
\end{tabular}

\begin{tabular}{|lccc|}
\hline \multicolumn{4}{|l}{ Tabla 3. Resultados del análisis multivariado de factores de riesgo para ITU/CUP } \\
\hline Variable & OR ajustado & IC 95 & Valor $\mathbf{p}$ \\
$\geq 80$ años & 0,928 & $0,462-1,863$ & 0,834 \\
$\geq 15$ días de hospitalización pre-CUP & 6,600 & $2,280-19,10$ & 0,001 \\
\hline Diabetes mellitus 2 & 0,711 & $0,327-1,543$ & 0,388 \\
Vejiga neurogénica & 0,277 & $0,052-1,468$ & 0,131 \\
$\geq 7$ días de CUP & 1,991 & $1,005-3,945$ & 0,048 \\
Cumple criterios de indicación de CUP & 1,368 & $0,685-2,780$ & 0,368 \\
\hline
\end{tabular}

ITU/CUP: infección del tracto urinario secundario a catéter urinario permanente, CUP: catéter urinario permanente.
CUP. Esta ventana de tiempo resultó ser algo mayor a lo identificado en la literatura científica, como es un estudio de cohorte que demostró riesgo al sexto día de hospitalización ${ }^{2,7}$. Nuestros resultados apuntan a una hospitalización prolongada como factor de riesgo per $s e$, lo cual es congruente con lo descrito por Piechota en un estudio caso-control australiano que identificó varios factores de riesgo para ITU/CUP incluyendo una estadía hospitalaria prolongada $>3$ meses $(\mathrm{p}<0,001)^{3}$.

\section{ITU/CUP y cumplimiento de los criterios de indicación de CUP}

El presente estudio no mostró diferencias significativas en la indicación de CUP entre casos y controles. La literatura científica señala que entre 21 y $54 \%$ de los pacientes no tiene una indicación adecuada para este dispositivo y un error frecuente es su instalación en casos de incontinencia urinaria sin una herida perianal o sacra ${ }^{7,16,21}$ que lo justifique. Holroyd-Leduc y cols. demostraron que los pacientes mayores de 70 años con comorbilidades y una mayor gravedad de la enfermedad y pacientes geriátricos, tenían un alto riesgo de cateterización urinaria sin una indicación médica adecuada, aumentando el riesgo de 
eventos adversos relacionados a la atención ${ }^{22}$. En un estudio chileno realizado en una UCI de alta complejidad encontraron que 19,2\% de los pacientes no cumplía con criterios de indicación de CUP ${ }^{23}$, de manera similar un trabajo brasileño mostró que la indicación inadecuada puede llegar a ser del $24 \%{ }^{24}$.

\section{ITU/CUP en pacientes con vejiga neurogénica}

Estudiamos esta comorbilidad en consideración a que la sobredistensión vesical es uno de los factores de riesgo más importantes de ITU. En nuestra investigación no encontramos diferencias significativas entre casos y controles, probablemente porque pocos pacientes usaron CUP por esta causa $(6,3 \%$ en casos y $7,3 \%$ en controles; $p$ $0,2)$. Si bien el cateterismo intermitente se ha considerado como el estándar de oro en el manejo de estos pacientes, es importante tener en cuenta que hay ciertos criterios tales como una capacidad de la vejiga menor a $200 \mathrm{ml}$ o un alto consumo o administración de fluidos, que hace necesaria la indicación de un cateterismo permanente. Además, se cuenta con el antecedente de que $80 \%$ de los pacientes con vejiga neurogénica prefieren el uso de dispositivo urinario permanente, puesto que hay pocas publicaciones que comparen entre si los distintos tipos de cateterismo para evitar distensión vesical en esta patología $^{25}$. No obstante, es preciso mencionar que un estudio español observó que había un mayor riesgo de infección con el CUP respecto a otras alternativas como un catéter suprapúbico, el cateterismo urinario intermitente o el uso de estuche peneal ${ }^{25-27}$.

\section{ITU/CUP y diabetes mellitus}

En nuestra experiencia, no se identificó la diabetes mellitus como factor de riesgo para ITU/CUP. Estos resultados son similares a lo descrito en estudios previos donde se encontró que niveles de glicemia mayor a 110 $\mathrm{mg} / \mathrm{dl}$ se asociaba con la presencia de bacteriemia asociada a CVC y sobre $180 \mathrm{mg} / \mathrm{dl}$ con neumonía asociada a ventilación mecánica, pero no con ITU/CUP ${ }^{6,28}$. En contraste, en estudios realizados en E.U.A y Canadá encontraron que el grupo de pacientes diabéticos constituía un factor de riesgo no modificable para ITU/CUP ${ }^{7,9,29}$.

\section{Edad e ITU/CUP}

Propusimos evaluar una edad $\geq 80$ años como factor de riesgo de acuerdo a la revisión bibliográfica que indica que ya sobre los 50 o 60 años se puede considerar como tal. En nuestra población estudiada los resultados no fueron significativos para esta variable, tampoco para una edad $\geq 50$ años. La edad fue identificada como un factor de riesgo en un estudio caso control en pacientes quirúrgicos donde la edad media de los pacientes que experimentaron una ITU postoperatoria fue mayor que aquellos que no la presentaron ${ }^{7}$. Asimismo, el aumento de la edad fue identificada como un factor de riesgo progresivo en el desarrollo de una ITU postoperatoria: 60-69 años (OR, 1,5; IC de 95\%, 1,4 a 2,9), 70-79 años (OR, 2,0 IC de $95 \%, 1,4$ a 2,9), $\geq 80$ años (OR, 2,3; IC de 95\%, 1,5 a $3,6)^{9}$. Puede ser que la diferencia de nuestros resultados con los comentados se deba al bajo número de pacientes quirúrgicos.

Algunas de las limitaciones que debemos mencionar de este estudio son la falta de análisis de otros factores de riesgo, tales como el cumplimiento de la técnica aséptica en la instalación del CUP y cumplimiento de las medidas de mantención del dispositivo, ya que no fue posible obtener estos datos en forma retrospectiva a través de la revisión del registro clínico. Tampoco se revisó el impacto de otros factores de riesgo conocidos como es el género, ya que se utilizó como criterio de pareo de casos y controles, ni tampoco se consideró el valor de la creatinina y el lugar de instalación del CUP. Pese a ello, nuestros resultados son significativos y útiles para delinear medidas de intervención con sustento científico, y enfatizan la importancia de disminuir la duración del CUP.

\section{Conclusiones}

En esta investigación se logró identificar dos factores de riesgo independientes para ITU/CUP, la duración del CUP $\geq 7$ días y la instalación del CUP con 15 días y más de hospitalización. En contrapunto, no se encontró una asociación con otros factores de riesgo descritos en la literatura especializada.

De acuerdo lo anterior, las medidas de prevención deben estar focalizadas en una indicación correcta de instalación de CUP en pacientes con más de dos semanas de hospitalización, para lo cual debe existir directrices claras que faciliten la toma de decisiones a nivel local, con la respectiva difusión y capacitación en el equipo de salud. En segundo término, se debe focalizar los esfuerzos en la evaluación diaria de la necesidad de mantención del CUP, para lo cual se sugiere implementar estrategias tecnológicas de recordatorios u otras, las que son de gran ayuda. Si bien los datos demuestran que a nivel local la indicación de instalación del dispositivo es adecuada, no existe una evaluación diaria de la necesidad del dispositivo lo cual hace que este se mantenga en el tiempo de manera innecesaria constituyendo un riesgo de ITU/CUP, es por ello que la monitorización de la duración y el retiro oportuno es uno de nuestros desafíos a corto plazo. 


\section{—}

\section{Referencias bibliográficas}

1.- Chen Y, Zhao J Y, Shan X, Han X L, Tian S G, Chen F Y, et al. A point-prevalence survey of healthcare-associated infection in 52 Chinese hospitals. J Hosp Infect 2017; 95: 105-11. doi: 10.1016/j.jhin.2016.08.010.

2.- Leone M, Albanèse J, Garnier F, Sapin C, Barrau K, Bimar M C, et al. Risk factors of nosocomial catheter-associated urinary tract infection in a polyvalent intensive care unit. Intensive Care Med 2003; 29: 1077-80. doi: 10.1007/s00134-003-1767-2.

3.- Piechota H. Prevention of catheter-associated urinary tract infections. Aktuelle Urol 2016; 47: 220-22. doi: 10.1055/s-0042-101845.

4.- Durdu B, Hakyemez I N, Bolukcu S, Okay G, Gultepe B, Aslan T. Mortality markers in nosocomial Klebsiella pneumoniae bloodstream infection. Springerplus 2016; 5: 1892. doi: 10.1186/s40064-016-3580-8.

5.- Otaiza F, Orsini M, Pholenz M. Informe Vigilancia Minsal 2017. Disponible en: https:// www.minsal.cl/wp-content/uploads/2015/09/ informe-vigilancia-2017.pdf. Fecha de acceso: 22 de septiembre de 2019.

6.- Bursle E C, Dyer J, Looke D F, McDougall D A, Paterson D L, Playford E G. Risk factors for urinary catheter associated bloodstream infection. J Infect 2015; 70: 585-91. doi: 10.1016/j.jinf.2015.01.001.

7.- Chenoweth C, Saint S. Preventing catheter associated urinary tract infections in the intensive care unit. Crit Care Clin 2013; 29 : 19-32. doi:10.1016/j.ccc.2012.10.005.

8.- Chenoweth CE, Saint S. Urinary tract infections. Infect Dis Clin North Am 2011; 25 : 103-15. doi: 10.1016/j.idc.2010.11.005.

9.- Alvarez A, Demzik A, Alvi HM, Hardt KD, Manning DW. Risk factors for postoperative urinary tract infections in patients undergoing total joint arthroplasty. Adv Orthop 2016: 2016:7268985. doi: 10.1155/2016/7268985.

10.- Vincitorio D, Barbadoro P, Pennacchietti L, Pellegrini I, David S, Ponzio E, et al. Risk factors for catheter-associated urinary tract infection in Italian elderly. Am J Infect Control 2014; 42: 898-901. doi: 10.1016/j. ajic.2014.05.006.

11.- Barbadoro P, Labricciosa FM, Recanatini C, Gori G, Tirabassi F, Martini E, et al. Catheterassociated urinary tract infection: Role of the setting of catheter insertion. Am J Infect Control 2015; 43: 707-10. doi: 10.1016/j. ajic.2015.02.011.

12.- Tsuchida T, Makimoto K, Ohsako S, Fujino M, Kaneda M, Miyazaki T, et al. Relationship between catheter care and catheter-associated urinary tract infection at Japanese general hospitals: a prospective observational study. Int J Nurs Stud 2008; 45: 352-61. doi:10.1016/j. ijnurstu.2006.10.006.

13.- Circular $\mathrm{C} 13 \mathrm{~N}^{\circ} 06$. Definiciones y Criterios de notificación de Infecciones Asociadas a la Atención en Salud (IAAS) para la vigilancia epidemiológica. Ministerio de Salud de Chile 2016. Disponible en: https://www. minsal.cl/wp-content/uploads/2017/01/ Manual-Definiciones-para-Sistema-deVigilancia-Epidemiol\%c3\%b3gica-IAAS-2017corregido-23-01-2017.pdf. Fecha de acceso: 22 de noviembre de 2019.

14.- Gould C V, Umscheid C A, Agarwal R K, Kuntz G, Pegues D A and the Healthcare Infection Control Practices Advisory Committee Guideline for Prevention of Catheter-Associated Urinary Tract Infections 2009. Last update: June 6, 2019: 1-61. Disponible en: https://www.cdc. gov/infectioncontrol/pdf/guidelines/cautiguidelines-H.pdf

15.- Savas L, Guvel S, Onlen Y, Savas N, Duran N. Nosocomial urinary tract infections: microorganisms, antibiotic sensitivities and risk factors. West Indian Med J 2006; 55: 188-93. doi: 10.1590/s0043-31442006000300011.

16.- Kim B, Pai H, Choi W S, Kim Y, Kweon K T, Kim H A, et al. Current status of indwelling urinary catheter utilization and catheterassociated urinary tract infection throughout hospital wards in Korea: A multicenter prospective observational study. PLoS One 2017; 12: e0185369. doi: 10.1371/journal. pone. 0185369 .

17.- Saint S, Wiese J, Amory J K, Bernstein M L, Patel U D, Zemencuk J K, et al. Are physicians aware of which of their patients have indwelling urinary catheters? Am J Med 2000; 109: 476-80. doi: 10.1016/s00029343(00)00531-3.

18.- van den Broek P J, Wille J C, van Benthem B H, Perenboom R J, van den Akker-van Marle M E, Niël-Weise BS. Urethral catheters: can we reduce use? BMC Urol 2011; 11: 10. doi: 10.1186/1471-2490-11-10.

19.- Bell M, Alaestante G, Finch C. A multidisciplinary intervention to prevent catheter-associated urinary tract infections using education, continuum of care, and Systemwide Buy-In. Ochsner J 2016 Spring; 16: $96-100$

20.- Meddings J, Rogers M A, Krein S L, Fakih M G, Olmsted R N, Saint S. Reducing unnecessary urinary catheter use and other strategies to prevent catheterassociated urinary tract infection: an integrative review. BMJ Quality \& Safety 2014; 23: 277-89. doi: 10.1136/bmjqs-2012-001774.

21.- Jansen I A, Hopmans T E, Wille J C, van den Broek P J, van der Kooi T, van Benthem B. Appropriate use of indwelling urethra catheters in hospitalized patients: results of a multicenter prevalence study. BMC Urol 2012; 12: 1-6. doi: 10.1186/1471-2490-12-25.

22.- Holroyd-Leduc J M, Sands L, Palmer R, Kresevic D, Landefeld CS. Risk factors for indwelling urinary catheterization among older hospitalized patients without a specific medical indication for catheterization. J Patient Saf 2005; 1: 201-7. doi: 10.1097/01. jps.0000205737.68588.d5.

23.- Gálvez R, Berasain M, Luengo C, Cifuentes M, Tobar E, Jemenao M, et al. Adherencia médica a las indicaciones de procedimientos invasores en la unidad de pacientes críticos de un hospital universitario. Rev Chilena Infectol 2014; 31: 528-33. doi: 10.4067/S0716-10182014000500003.

24.- de Oliveira L, Andrade J, Masson W. Uso excessivo do cateter vesical em pacientes internados em enfermarias de hospital universitário. Rev Esc Enferm USP 2011; 45: 1089-96. doi: 10.1590/S008062342011000500009 .

25.- Linsenmeyer T A. Catheter-associated urinary tract infections in persons with neurogenic bladders. J Spinal Cord Med 2018; 41: 132-41. doi: 10.1080/10790268.2017.1415419.

26.- Bonfill X, Rigau D, Esteban-Fuertes M, Barrera-Chacón J M, Jáuregui-Abrisqueta M L, Salvador S, et al; ESCALE Study Group. Efficacy and safety of urinary catheters with silver alloy coating in spinal cord injured patients: a multicentric pragmatic randomized controlled trial. The ESCALE trial. Spine J 2017; 17: 1650-1657. doi: 10.1016/j. spinee.2017.05.025.

27.- Esclarín de Ruz A, García Leoni E, Herruzo Cabrera R. Epidemiology and Risk factor for urinary tractus infeccion in patients with spinal cord injure. J Urol 2000; 164: 1285-9. doi: 10.1016/S00225347(05)67157-1.

28.- Jeon C, Furuya E Y, Smaldone A, Larson E L. Post-admission glucose levels are associated with healthcare-associated bloodstream infections and pneumonia in hospitalized patients with diabetes. J Diabetes Complications 2012; 26: 517-21. doi:10.1016/j. jdiacomp.2012.06.007.

29.- Nicolle L E. Urinary catheter-associated infections. Infect Dis Clin North Am 2012; 26:13-27. doi: 10.1016/j.idc.2011.09.009. 